Meta

Journal des tradlucteurs

Translators' Journal

\title{
Peut-on traduire les civilisations ?
}

\section{Naïm Kattan}

Volume 27, numéro 4, décembre 1982

URI : https://id.erudit.org/iderudit/003287ar

DOI : https://doi.org/10.7202/003287ar

Aller au sommaire du numéro

Éditeur(s)

Les Presses de l'Université de Montréal

ISSN

0026-0452 (imprimé)

1492-1421 (numérique)

Découvrir la revue

Citer cet article

Kattan, N. (1982). Peut-on traduire les civilisations ? Meta, 27(4), 401-403.

https://doi.org/10.7202/003287ar d'utilisation que vous pouvez consulter en ligne.

https://apropos.erudit.org/fr/usagers/politique-dutilisation/ 


\section{PEUT-ON TRADUIRE LES CIVILISATIONS ?*}

NAÏM KATTAN

Avant sa mort, le critique Adam Tarn préparait un livre sur Tchékhov. Traducteur de Beckett et de Ionesco en polonais, Tarn publiait à Varsovie une revue théâtrale Dialogue, avant d'être obligé de chercher refuge au Canada. La mort le surprit avant d'avoir terminé son grand projet: une redécouverte du vrai Tchékhov, une réhabilitation de son œuvre. Tchékhov est un écrivain comique me dit-il un soir, me faisant part de son entreprise. En Occident, on l'a transformé en porte-drapeau de la nostalgie, ou on en a fait un mélancolique. Il suffirait de lire ses lettres, ses notes de mise en scène pour se rendre compte qu'il était un écrivain comique.

-- Il se voulait comique, cherchais-je à rectifier. Un écrivain n'est pas toujours le meilleur juge de son oeuvre.

J'ai oublié la suite de l'entretien et pourtant j'y pense chaque fois que je lis ou relis une ouvre russe. En Occident, cette littérature a une existence propre. Nombreux sont les écrivains qui ont subi l'influence de Dostoïevski et de Tolstoï. Rares sont ceux parmi eux qui connaissaient le russe ou qui l'ont appris. Aussi le grand roman russe du dix-neuvième siècle existe en anglais et en français indépendamment de la substance originelle. Les points de comparaison entre l'œuvre originale et le texte traduit n'existent presque pas. Il y a par conséquent une littérature russe en traduction, autonome, séparée de celle, originale dont elle est issue.

Ce n'est pas le cas de Shakespeare malgré les nombreuses et les grandes traductions auxquelles son œuvre a donné lieu. Peut-être est-elle tellement immense qu'elle échappe à toutes les réductions. Peut-être est-ce aussi en raison de la langue dans laquelle elle est écrite, l'anglais étant depuis longtemps la langue seconde de tant de pays et de tant de personnes. Cependant, une telle affirmation ne peut être entièrement vraie car le français, langue elle aussi éminemment universelle, n'a pas fait de Corneille ou de Racine des écrivains mondiaux. Serait-ce que leurs oeuvres soient récalcitrantes aux traductions? Peut-être aussi Shakespeare est-il, par rapport à la civilisation britannique, à la fois l'expression de ce qu'elle est et de ce qui la dépasse tandis que Racine et Corneille ne traduisent ce qui dépasse la civilisation française que dans la mesure où ils l'expriment.

Il y a quelques années $j$ 'écrivis une pièce sur le rapport des frères, en l'occurrence Jacob et Esaü. Relisant la Bible, je me rendis compte que ce qui

* Colloque de Glendon, 1980. 
fut traduit en anglais et en français et sans doute dans d'autres langues en droit d'aînesse n'est en hébreu qu'aînesse. Ce n'est donc ni un concept ni une notion juridique. C'est un fait, une chose. Cette absence de distance entre le mot et la chose, le concept et le concret, est confirmé par le mot davar qui veut dire à la fois mot et chose. Mais allons plus loin et prenons un autre mot: avon. Pendant des siècles, ce mot fut traduit par péché. Or il s'agit en fait de tort. Cela indique une distance entre deux approches au réel, deux comportements, deux attitudes morales. En un mot nous sommes en face de deux civilisations. L'une considère le avon comme un tort, un fait concret donc réparable et l'autre comme un manquement moral qui renvoie à la culpabilité.

Prenons maintenant un autre mot, un mot arabe cette fois: Djihad. On l'a traduit depuis des siècles par guerre sainte. Et cette fois nous sommes en pleine réalité car Khomeini lance périodiquement des appels au Djihad, à la guerre sainte. Et pourtant, il n'y a dans le mot ni la notion de guerre ni celle de sacralité. Une traduction possible pourrait être l'effort. Et un mot dérivé d'une même racine Ijtihad désigne l'effort dans l'étude et, historiquement désignait plus particulièrement l'étude interprétative du Coran. En réalité Djihad signifie en fin de compte guerre sainte mais on parvient à cette réalité par d'autres voies. Il est question d'un effort religieux, d'un travail ardu en faveur de Dieu. Cela finit par être une guerre et pour l'occidental une guerre qualifiée, une guerre sainte. Car pour l'Occident, la frontière est bien tracée entre le sacré et le profane. Dans une civilisation où cette notion n'existe pas ou n'existe pas autant la guerre sainte n'est qu'une extension d'un effort religieux, d'un ardent travail en faveur de Dieu.

La parole de Dieu étant intraduisible, les Musulmans, qu'ils soient Turcs ou Indonésiens, doivent prier et lire le Coran en arabe, langue qu'ils ne connaissent ni ne comprennent. Le message n'est pas transmissible dans une langue autre que celle de l'origine. Dans ce cas précis, une civilisation, du moins dans une de ses essentielles dimensions, n'est pas traduisible. La langue, rapport primordial avec le réel est globalisante et tient ses adeptes captifs, tels des sujets obéissants. Ils réagissent à partir de l'émotion qu'elle éveille à la politique et à l'histoire. Ici le cliché rejoint le tréfond de l'émotion. On peut aller très loin, bien que le chemin soit hasardeux, et dire que les Arabes ont du mal à vivre avec leur minorité qu'elle soit kurde ou copte, leur langue leur ayant donné des habitudes totalisantes. Car, en fait, accepter la traduction c'est admettre volontairement que dans le processus de transmission, il $y$ ait perte et abandon. Une dimension de cette civilisation est oblitérée, altérée afin qu'elle soit et compréhensible et acceptable à une autre. Des deux côtés, du point de départ au point d'arrivée, il y a reconnaissance d'un abandon de la totalisation. Djihad n'est pas guerre sainte. Pourtant si on est occidental, si on parle le français ce ne peut être que guerre sainte. Autrement il faut apprendre l'arabe et à travers cette langue pénétrer une civilisation et une histoire autrement dit se transformer en anthropologue. Or il y a dans cet idéal de compréhension de l'autre, de pénétration de l'étranger un danger; c'est celui de tout réduire à un sujet d'étude et d'être ainsi incapable de prendre des positions morales et éthiques, c'est-à-dire d'appartenir à une civilisation. 
Une civilisation est le désir qu'un individu vit au-delà du temps et de l'espace. Elle ne peut survivre à l'enfermement. Elle se nourrit de l'échange. Et tout échange est l'acceptation d'une perte de soi, pour acquérir chez l'autre, dans son être et sa substance, un élément de renouvellement. Qu'il y ait un écart entre péché et avon n'a de sens qu'historique. L'Occident a puisé dans l'Orient premier juif puis chrétien les éléments qui lui ont permis de bâtir sa civilisation. Et notre jugement vis-à-vis cette civilisation ne peut être une étude sur sa fidélité aux origines, sur son authenticité et sur sa loyauté envers les emprunts. On ne peut la juger qu'en fonction de la possibilité qu'elle a donnée et qu'elle donne à deś hommes et à des femmes de vivre le plus pleinement possible leur désir. Par conséquent cela donne lieu à des ambiguïtés et à des contradictions. Cela permet, par l'acceptation de l'abandon, par l'admission de l'autre, d'échapper à l'enfermement de la totalisation et de respirer un air de liberté. En traduisant avon par péché l'Occident a emprunté ce qu'il voulait, ce dont il avait besoin, ce qu'il pouvait admettre. Il a utilisé cette notion comme moyen de contrôle de son désir. Il aurait pu l'inventer ou choisir une autre. Seule était possible, pour la transmission d'une civilisation à une autre, une mauvaise traduction, non une perte de sens, mais sa modification, son altération, pour que ce sens reste vivant à la réception, au point d'arrivée. Autrement il se serait agi d'une oeuvre d'anthropologie, d'un travail d'étude historique. Si une civilisation est un texte il faut y échapper pour la traduire, inventer un second à partir de l'original, le récrire certes, mais aussi découvrir des mots neufs à partir des signes reconnus. La nouvelle écriture n'est ni l'une ni l'autre des deux civilisations mais l'une modifiée par l'autre, remodelée, réinventée. Pour le meilleur et le pire, c'est une civilisation autre.

Il n'est pas toujours question d'échange. La traduction peut aussi être une tentative de compréhension afin d'ajuster un comportement, de s'adapter à ce qui apparaît comme une étrangeté. L'exactitude est alors requise. Comprendre l'autre d'abord. Décider de le figer dans son étrangeté, transformer la différence en territoire inviolable interdisant l'altération, la modification. La porte est close à l'échange. La traduction est alors œuvre d'exégèse qui laisse le receveur dans son monde, inaltérable, indifférent. Le monde de l'autre est singulier. Tout y est permis. On observe, on enregistre. On n'a ni à comprendre, ni à accepter. Devant la pureté de l'autre, on conserve la sienne.

Une autre forme de traduction est le dépouillement d'une œuvre de son arrière-plan originel pour la doter d'un arrière-plan d'emprunt. Transplanter le squelette d'une oeuvre et l'adapter à la langue de réception est une autre forme de fermeture, une négation de la différence.

L'ouvre appartient à une civilisation. L'ignorer, l'en détacher, tenter de la doter d'une civilisation d'emprunt c'est ignorer son irréductible individualité. Une telle réduction n'ajoute rien à la langue de réception puisque c'est un corps infirme, un corps amputé qui est proposé et non un corps vivant, entier, dans son originalité première. 